\title{
Tracer input for kinetic modelling of liver physiology determined without sampling portal venous blood in pigs
}

\author{
Michael Winterdahl • Susanne Keiding • \\ Michael Sørensen • Frank Viborg Mortensen • \\ Aage Kristian Olsen Alstrup • Ole Lajord Munk
}

Received: 26 May 2010/Accepted: 3 September 2010 / Published online: 30 September 2010

(C) The Author(s) 2010. This article is published with open access at Springerlink.com

\begin{abstract}
Purpose Quantification of hepatic tracer kinetics by PET requires measurement of tracer input from the hepatic artery (HA) and portal vein (PV). We wished to develop a method for estimating dual tracer input without the necessity to sample PV blood.

Methods Pigs weighing $40 \mathrm{~kg}$ were given bolus doses of $\mathrm{C}^{15} \mathrm{O}(\mathrm{CO}), 2-\left[{ }^{18} \mathrm{~F}\right]$ fluoro-2-deoxy-D-glucose (FDG), $\left[{ }^{11} \mathrm{C}\right]-$ methylglucose (MG), 2-[ $\left.{ }^{18} \mathrm{~F}\right]$ fluoro-2-deoxy-D-galactose (FDGal) or $\mathrm{H}_{2}{ }^{15} \mathrm{O}\left(\mathrm{H}_{2} \mathrm{O}\right)$. Tracer concentration 3-min time courses were measured in the femoral artery and PV by blood sampling. Blood flow was measured in the HA and PV using flow-meters. A model for transfer of tracer through the splanchnic circulation was used to estimate values of a tracer-specific model parameter $\beta$. Tracerspecific mean values of $\beta$ were used to estimate tracer concentration time courses in the PV from the measured arterial concentration. A model-derived dual-input was calculated using the mean HA flow fraction $(0.25)$ and validated by comparison of the use of the measured dualinput and a kinetic model with a fixed "true" $K_{1}^{\text {true }}$, i.e. clearance of tracer from blood to liver cells.
\end{abstract}

M. Winterdahl $\cdot$ S. Keiding $(\bowtie) \cdot$ M. Sørensen •

A. K. O. Alstrup • O. L. Munk

PET Centre, Aarhus University Hospital,

DK-8000 Aarhus C, Denmark

e-mail: susanne@pet.auh.dk

S. Keiding $\cdot$ M. Sørensen

Department of Hepato-Gastroenterology V,

Aarhus University Hospital,

DK-8000 Aarhus C, Denmark

F. V. Mortensen

Department of Surgery L,

Aarhus University Hospital,

DK-8000 Aarhus C, Denmark
Results The rank order of the means of $\beta$ was $\mathrm{CO}<\mathrm{FDG} \approx$ $\mathrm{MG}<\mathrm{FDGal}<\mathrm{H}_{2} \mathrm{O}$, reflecting their different splanchnic mean transit times. Estimated $K_{1}{ }^{\text {est }}$ was not significantly different from "true" $K_{1}{ }^{\text {true }}$.

Conclusion The hepatic dual tracer input, which is of great importance for the assessment of processes such as transfer across the plasma-hepatocyte membrane or hepatic blood perfusion, can be well approximated in pigs without the necessity to sample PV blood and measure hepatic blood flow; only arterial blood sampling is needed.

Keywords Splanchnic circulation - Liver kinetics ·

Molecular imaging · Pharmacokinetics · PET · Portal model · Radioactive tracers

\begin{tabular}{|c|c|}
\hline \multicolumn{2}{|c|}{ Abbreviations } \\
\hline $\mathrm{CO}$ & {$\left[{ }^{15} \mathrm{O}\right]$-Carbon monoxide } \\
\hline FDG & $2-\left[{ }^{18}\right.$ F]Fluoro-2-deoxy-D-glucose \\
\hline MG & {$\left[{ }^{11} \mathrm{C}\right]$ Methylglucose } \\
\hline FDGal & $2-\left[{ }^{18}\right.$ F $]$ Fluoro-2-deoxy-D-galactose \\
\hline $\mathrm{H}_{2} \mathrm{O}$ & {$\left[{ }^{15} \mathrm{O}\right]$-Water } \\
\hline HA & Hepatic artery \\
\hline PV & Portal vein \\
\hline$F_{\mathrm{HA}}$ & Blood flow in the hepatic artery $(\mathrm{ml} \mathrm{blood} / \mathrm{min})$ \\
\hline$F_{\mathrm{PV}}$ & Blood flow in the portal vein $(\mathrm{ml}$ blood $/ \mathrm{min})$ \\
\hline$f_{\mathrm{HA}}$ & Hepatic arterial blood flow fraction \\
\hline$\overline{f_{\mathrm{HA}}}$ & Mean $f_{\mathrm{HA}}$ for all experiments \\
\hline$h(t)$ & $\begin{array}{l}\text { Impulse-response function of the portal vein } \\
\text { model }\end{array}$ \\
\hline$\beta$ & $\begin{array}{l}\text { Tracer-specific parameter of the portal vein } \\
\text { model (min) }\end{array}$ \\
\hline $\bar{\beta}$ & Population mean of $\beta$ for each tracer (min) \\
\hline$C_{\mathrm{A}}(t)$ & $\begin{array}{l}\text { Measured tracer concentration time-course } \\
\text { in peripheral arterial blood }(\mathrm{kBq} / \mathrm{ml} \text { blood })\end{array}$ \\
\hline$C_{\mathrm{HA}}(t)$ & Tracer concentration time course in hepatic \\
\hline
\end{tabular}


arterial blood $(\mathrm{kBq} / \mathrm{ml}$ blood);

tracer concentration is equal to that in $C_{\mathrm{A}}(t)$

$C_{\mathrm{PV}}(t) \quad$ Measured tracer concentration time course

in the portal vein $(\mathrm{kBq} / \mathrm{ml}$ blood $)$

$\widetilde{C}_{\mathrm{PV}}(t) \quad \mathrm{PV}$ model-derived tracer concentration time course in the portal vein $(\mathrm{kBq} / \mathrm{ml}$ blood)

$C_{\text {dual }}(t) \quad$ Measured dual-input tracer concentration time course $(\mathrm{kBq} / \mathrm{ml}$ blood $)$

$\widetilde{C}_{\text {dual }}(t)$ Model-derived dual-input tracer concentration time course $(\mathrm{kBq} / \mathrm{ml}$ blood $)$

$C_{\text {liver }}(t) \quad$ Measured activity concentration time course in liver tissue $(\mathrm{kBq} / \mathrm{ml}$ liver tissue versus min)

$\widetilde{C}_{\text {liver }}(t) \quad$ Simulated activity concentration time course in liver tissue $(\mathrm{kBq} / \mathrm{ml}$ liver tissue versus min)

$K_{1} \quad$ Clearance of tracer from blood to liver cells ( $\mathrm{ml} \mathrm{blood} / \mathrm{ml}$ liver tissue/min)

\section{Introduction}

Molecular imaging with positron emission tomography (PET) provides unique possibilities for in vivo quantification of blood perfusion, transmembrane transport and tissue metabolism. A quantitative PET study comprises bolus administration of a positron-emitting tracer immediately followed by dynamic recording of the tracer concentration time-courses in tissue by PET and in the blood supply by blood sampling. Physiological parameters are calculated by fitting a mathematical model of tracer distribution and metabolism to the data. Application of this approach to the liver is complicated, however, due to dual input of tracer from the hepatic artery (HA) and the portal vein (PV), comprising $25 \%$ and $75 \%$, respectively. The tracer concentration time-course in the PV is initially delayed and dispersed compared to that in the HA due to transfer of tracer through the splanchnic circulation. If there is no metabolism of the tracer in the organs drained by the PV, hepatic steady-state metabolism may be assessed by dynamic PET using solely the arterial input of tracer. This has been validated for $2-\left[{ }^{18} \mathrm{~F}\right]$ fluoro-2-deoxy-D-glucose (FDG) $[1,2]$ and 2-[ $\left[{ }^{18} \mathrm{~F}\right]$ fluoro-2-deoxy-D-galactose (FDGal) in pigs [3] and used in studies of hepatic FDG metabolism in humans [4-6]. Processes such as transfer across the highly permeable plasma-hepatocyte membrane or hepatic blood perfusion must be assessed using the initial dynamic part of tracer concentration time-courses before steady-state metabolism dominates the recordings, however. This requires successive measurement of blood tracer activity concentration in the PV immediately after tracer administration, as well as of the fractional blood flow in the PV and HA [1]. The most reliable method is to measure tracer concentration time-courses in both an artery and the
PV by invasive blood sampling combined with invasive measurements of blood flow in the HA and PV. This is only possible in animal studies, however [1-3, 7, 8], and various studies have addressed the challenge of how to assess the initial dynamic tracer input from the HA and PV for human studies. Choi et al. [4] suggested the use of a single arterial input with a time delay to account for the delay of the PV input, but this does account for the dispersion of the PV tracer input. However, Munk et al. [1] showed that knowledge of the tracer input from both vessels, the socalled dual-input, is needed to obtain unbiased kinetic parameter estimates that are in agreement with independent measurements of hepatic blood flow and hepatic blood volume.

PET image-derived methods have been used in several studies [9-12] and seem attractive because they are completely noninvasive and thus applicable to humans. However, the image-derived dual-input tracer concentration time-course is prone to errors caused by partial volume effects where the tracer concentration time-courses in the $\mathrm{PV}$ and the aorta, used as arterial input, are affected by tracer activity in the surrounding tissue due to limited spatial resolution compared with the size of the vessels and respiratory motion $[4,9,10]$. Mathematical-physiological models describing the transfer of tracer from the intestinal arteries to the PV do not rely on the dynamic tissue PET image data. Various mathematical PV models have been proposed such as a one-compartmental model [13] and a double gamma-variate model [8].

We have previously developed PV models that included only parameters with direct physiological interpretations and which were validated by means of sets of measured tracer concentration time-courses in arterial blood and the PV [14]. However, these models have never been used to calculate the dual-input for kinetic modelling of liver physiology. One of these PV models (method 4 in ref. 14) is particularly useful and robust for calculating the modelderived tracer concentration time-course in the PV from that in arterial blood as it provides a full description of the tracer concentration time-course in the PV using only a single tracer-specific parameter, $\beta$, which reflects tracerspecific mean splanchnic transit time for the tracer from the intestinal arteries to the PV. Therefore we tested the use of this PV model in the present study.

The aim of the present study was to estimate population means of $\beta$ for five tracers with a wide range of mean splanchnic transit times and to calculate the dual input from the tracer-specific mean values of $\beta$ and an estimated mean of the HA blood flow fraction. The model-derived dual input was validated by fitting a simple kinetic model to a simulated tracer concentration time-course in liver tissue. We chose clearance of tracer from blood to liver cells, $K_{1}$, as our test parameter because the $K_{1}$ estimate is particularly 
sensitive to the initial dynamic shape of the dual-input curve [1]. Thus any errors produced by using the PV model in kinetic modelling will be reflected in the estimated $K_{1}$. Moreover, $K_{1}$ for MG, FDG and FDGal may be used as a measure of hepatic blood perfusion $[1,3]$. The overall aim of the study was to develop and validate a simple method based solely on blood sampling from a peripheral artery, tracer-specific means of $\beta$ and an estimated mean HA blood flow fraction - with which to estimate the combined tracer input to the liver from the HA and PV for use in PET studies of initial hepatic tracer kinetics.

\section{Materials and methods}

The investigations were performed in nine pigs given $\mathrm{C}^{15} \mathrm{O}$ (CO) by inhalation. Five of the pigs also received intravenous injections of $\mathrm{H}_{2}{ }^{15} \mathrm{O}\left(\mathrm{H}_{2} \mathrm{O}\right)$. In addition, the study included analysis of raw data from previously published studies in six pigs which each received intravenous injections of FDG and $\left[{ }^{11} \mathrm{C}\right]$ methylglucose (MG) [1], and ten pigs which received intravenous injections of FDGal [3]. In each study the tracer concentration timecourse was measured simultaneously in a femoral artery and the PV, and blood flow was measured in the HA and PV for the first 3 min following tracer administration using surgically placed ultrasound transit time flow-meters.

All studies were approved by the Danish Ministry of Legal Affairs and performed in accordance with European Union, national, and institutional guidelines for animal welfare.

\section{Experimental procedures}

Female pigs (Danish Landrace and Yorkshire cross-breed; body weight $38-42 \mathrm{~kg}$ ) were fasted for $16 \mathrm{~h}$ with free access to water. Anaesthesia was maintained by a constant intravenous infusion of $240 \mathrm{mg}$ propofol, $200 \mathrm{mg}$ S-ketamine and $40 \mathrm{mg}$ midazolam per hour and the animal was ventilated with air/ oxygen 2.2:1 by mechanical respiration. Catheters were inserted into a femoral artery (blood sampling) and a femoral vein (intravenous administration). The abdomen was opened and ultrasound transit-time flow-meter probes $(12 \mathrm{~mm}$ and 4 or $6 \mathrm{~mm}$, respectively; CardioMed; In vivo Aps, Norway) were positioned around the PV and HA (in some cases two HA vessels) for continuous measurement of blood flow [15, 16]. A 5.3F polyethylene catheter (William Cook, Denmark) was inserted directly into the PV for blood sampling; it was placed downstream of the flow-meter probe so as not to disturb the flow measurements. Physiological parameters were maintained as follows: body temperature $38.5-39.5^{\circ} \mathrm{C}$, arterial $p \mathrm{CO}_{2} 3.5-7.0 \mathrm{kPa}, p \mathrm{O}_{2} 18.0-25.0 \mathrm{kPa}, \mathrm{pH} 7.35-$ 7.45 and blood glucose $5.0-6.7 \mathrm{mmol} / \mathrm{l}$. The experiments were terminated by intravenous injection of $4 \mathrm{~g}$ pentobarbital sodium.

Tracer administration and blood concentration measurements

$\mathrm{C}^{15} \mathrm{O}(400 \mathrm{MBq})$ was administered as a 10 -s inhalation via the respirator; $\mathrm{H}_{2}{ }^{15} \mathrm{O}(300 \mathrm{MBq})$ was administered as a 15 -s intravenous injection. To assess reproducibility, these tracers were administrated twice in each pig. Starting at the beginning of tracer administration, arterial blood was drawn from the catheter in the femoral artery by an automated blood sampling apparatus and the blood concentration of tracer determined every $0.5 \mathrm{~s}$ (Allogg, Mariefred, Sweden), correcting for delay and dispersion in the sampling catheters [17]. In order not to disturb blood flow in the HA, the samples were collected from a femoral artery (the concentration of tracer in the femoral artery is identical to that in the HA; Keiding, unpublished observations). PV blood samples $(1 \mathrm{ml})$ were collected manually from the PV catheter every 5 th second 18 times and then every 15th second 6 times ( $3 \mathrm{~min}$ ). Tracer concentrations in blood $(\mathrm{kBq} / \mathrm{ml})$ were measured using a well counter (Packard Instruments, Meriden, CT). All tracer concentration measurements were corrected for radioactive decay back to the start of tracer administration.

With the FDG [1], MG [1] and FDGal [3] studies, tracer concentrations were measured in successive blood samples drawn manually from the femoral artery and PV over a period of 45-90 min. However, in the present study only measurements from the initial 3 minutes were used. For none of the five tracers were metabolites detectable in the blood $[1,3]$ and accordingly the measured tracer activity concentrations in the blood were used directly in the calculations.

Model considerations and calculations

With each administration of tracer the following calculations were performed:

1. The HA blood flow fraction, $f_{\mathrm{HA}}$, was calculated from the flow-meter measurements of blood flow in the HA, $F_{\mathrm{HA}}$ (millilitres blood per minute), and in the $\mathrm{PV}, F_{\mathrm{PV}}$ (millilitres blood per minute), as

$f_{\mathrm{HA}}=F_{\mathrm{HA}} /\left(F_{\mathrm{HA}}+F_{\mathrm{PV}}\right)$

The $f_{\mathrm{HA}}$ in individual experiments deviated by less than $1 \%$ per minute, and an individual mean value of $f_{\mathrm{HA}}$ was used in the individual experiments (Section 2, below). For the calculations of the model-derived dual-input, $\widetilde{C}_{\mathrm{PV}}(t)$, a mean value for all experiments, denoted $\overline{f_{\mathrm{HA}}}(=0.25 \pm 0.07$, mean $\pm \mathrm{SEM}$ ), was used (Section 5, below). 
2. The measured dual-input, $C_{\text {dual }}(t)$, was calculated from the tracer concentration time-courses in HA blood, $C_{\mathrm{HA}}(t)$, equivalent to the individually measured tracer concentration time-courses in arterial blood, $C_{\mathrm{A}}(t)$, and PV blood, $C_{\mathrm{PV}}(t)$, and $f_{\mathrm{HA}}$, as

$C_{\text {dual }}(t)=f_{\mathrm{HA}} C_{\mathrm{A}}(t)+\left(1-f_{\mathrm{HA}}\right) C_{\mathrm{PV}}(t)$

3. We describe the passage of a bolus from the arterial system through the splanchnic circulation to the PV using a previously validated impulse response function based on a distribution of washout rate constants. The full impulse response function has two parameters: a final washout rate constant, $k_{0}$, and $\beta$, that determine the mean transit time and the standard deviation of transit times for the passage of tracer from the intestinal arteries to the PV [14]. Thus, the model parameters have a clear physiological interpretation. In the limit of a slow final washout constant, $k_{0} \rightarrow 0$, the full impulse-response function is reduced to a power law function with the single parameter $\beta$. This one-parameter impulse response function provided a robust description of the tracer concentration time-course in the PV for CO and MG [14]. In the present study we accordingly estimated $\beta$ of this PV model for each tracer administration by nonlinear regression analysis of the relationship between $C_{\mathrm{PV}}(t)$ and $C_{\mathrm{A}}(t)$ :

$C_{\mathrm{PV}}(t)=\int_{0}^{t} h(t-\tau) C_{\mathrm{A}}(\tau) d \tau ; \quad h(t)=\beta /(t+\beta)^{2}$.

With $\mathrm{CO}$ and $\mathrm{H}_{2} \mathrm{O}$, agreement between the double determinations of $\beta$ was good (see Results) and the mean value for each animal was used.

4. The mean of $\beta$, denoted $\bar{\beta}$, was used as an estimate of a population mean of $\beta$ for each tracer and used to calculate individual PV model-derived tracer concentration time courses in the PV, $\widetilde{C}_{\mathrm{PV}}(t)$, from $C_{\mathrm{A}}(t)$ as:

$\widetilde{C}_{\mathrm{PV}}(t)=\int_{0}^{t} h(t-\tau) C_{\mathrm{A}}(\tau) d \tau ; \quad h(t)=\bar{\beta} /(t+\bar{\beta})^{2}$

5. The model-derived dual-input, $\widetilde{C}_{\text {dual }}(t)$, was calculated from $C_{\mathrm{A}}(t), \widetilde{C}_{\mathrm{PV}}(t)$ and $\overline{f_{\mathrm{HA}}}$, as:

$\widetilde{C}_{\text {dual }}(t)=\overline{f_{H A}} C_{A}(t)+\left(1-\overline{f_{H A}}\right) \widetilde{C}_{P V}(t)$

6. The use of $\widetilde{C}_{\text {dual }}(t)$ instead of $C_{\text {dual }}(t)$ when estimating hepatic tracer kinetics was assessed by means of individual simulated tracer activity concentration time courses in liver tissue, $\widetilde{C}_{\text {liver }}(t)$, using the first 3 minutes of the measured $C_{\text {dual }}(t)$ and a one-tissue compartmental model with a fixed set of "true" parameters: $K_{1}^{\text {true }}$, clearance of tracer from blood to liver cells $(1 \mathrm{ml}$ blood per millilitre liver tissue per minute); $k_{2}$, rate constant for backflux of tracer from cells to blood $(2 / \mathrm{min})$; and $V_{0}$, vascular volume of the liver $(0.40 \mathrm{ml}$ blood per millilitre liver tissue). The model was fitted to $\widetilde{C}_{\text {liver }}(t)$ using the $\widetilde{C}_{\text {dual }}(t)$ as input for estimation of $K_{1}{ }^{\text {est }}$ with no parameters fixed. The error associated with the use of $\widetilde{C}_{\text {dual }}(t)$ rather than $C_{\text {dual }}(t)$ in the kinetic fitting procedure was assessed by the relative deviation of $K_{1}^{\text {est }}$ from $K_{1}^{\text {true }}:\left(K_{1}{ }^{\text {est }}-K_{1}{ }^{\text {true }}\right) / K_{1}{ }^{\text {true }}$, which assesses how well the fixed $K_{1}{ }^{\text {true }}$ is recovered. Although the one-tissue compartmental model was not physiologically correct for all tracers in the present study, we used it to generate a simulated tissue curve for testing the effect of replacing $C_{\text {dual }}(t)$ by $\widetilde{C}_{\text {dual }}(t)$ in the analysis of initial hepatic tracer kinetics.

\section{Statistical analysis}

Normally distributed data are expressed in terms of the mean \pm standard error of the mean (SEM) unless otherwise stated, and statistical analysis of intergroup differences was performed by ANOVA analysis. For multiple comparisons, the Bonferroni post-hoc test was used for data with equal variance and the Tamhane $\mathrm{T} 2$ test for data with unequal variance. Differences with a $p$ value $<0.05$ were considered statistically significant.

\section{Results}

Examples of the measured tracer concentration time-course in the PV, $C_{\mathrm{PV}}(t)$, are shown in Fig. 1 (left) which illustrates the delay and the dispersion of the peak compared to that in arterial blood, $C_{\mathrm{A}}(t)$. The $\widetilde{C}_{\mathrm{PV}}(t)$ derived from the $\mathrm{PV}$ model (Eq. 4) well reflects the measured $C_{\mathrm{PV}}(t)$.

With CO (nine tracer administrations) the degree of agreement between the double determinations of $\bar{\beta}$, expressed as an intraclass correlation, was 0.90 . The corresponding figure for $\mathrm{H}_{2} \mathrm{O}$ (five tracer administrations) was 0.86 . Thus, the estimate of $\beta$ could be accurately reproduced in each animal. For the administration of both FDG and MG in each of six pigs two-way ANOVA showed that the variation between the pigs accounted for $79.0 \%$ of the total variance $(p=0.0591)$. In other words, the accuracy of the estimated $\beta$ was good enough to demonstrate variation between the animals. The variation associated with $\bar{\beta}$ accordingly can be ascribed to physiological and experimental differences between animals which contribute to the error of $\widetilde{C}_{\mathrm{PV}}(t)$. The tracer-specific means of $\bar{\beta}$, are shown in Table 1 . The rank order for $\bar{\beta}$ was $\mathrm{CO}<\mathrm{FDG} \approx \mathrm{MG}<\mathrm{FDGal}<\mathrm{H}_{2} \mathrm{O}$.

As illustrated in Fig. 1 (right), the dual input, $\tilde{C}_{\text {dual }}(t)$, derived from the model (Eq. 5) well reflects the measured dual input, $C_{\text {dual }}(t)$ (Eq. 2). $\widetilde{C}_{\text {dual }}(t)$ even reproduced the biphasic shape of $C_{\text {dual }}(t)$ for CO. In some cases, $\widetilde{C}_{\text {dual }}(t)$ 
Fig. 1 Left Examples of blood tracer activity concentration time-courses following bolus administration of tracers to pigs measured in a femoral artery, $C_{\mathrm{A}}(t)$ (red curves); measured in the $\mathrm{PV}, C_{\mathrm{PV}}(t)$ (blue curves); $\mathrm{PV}$ model-derived, $\widetilde{C}_{\mathrm{PV}}(t)$ (green curves) calculated from $C_{\mathrm{A}}(t)$ using means of the tracerspecific parameters for the passage of the tracer from the intestinal arteries to the PV, $\bar{\beta}$ s (Eq. 4). Right For each tracer, the measured dual input, $C_{\text {dual }}(t)$ (blue curves), was calculated from $C_{\mathrm{A}}(t)$ and $C_{\mathrm{PV}}(t)$ shown on the left and the individually measured HA blood flow fractions, $f_{\mathrm{HA}}$ (Eqs. 1 and 2). The corresponding model-derived dual input, $\widetilde{C}_{\text {dual }}(t)$ (green curves), was calculated from $C_{\mathrm{A}}(t)$ and $\widetilde{C}_{\mathrm{PV}}(t)$ and the mean HA blood flow fraction, $f_{\mathrm{HA}}$ $(=0.25$; Eq. 5)
Artery and Portal Vein
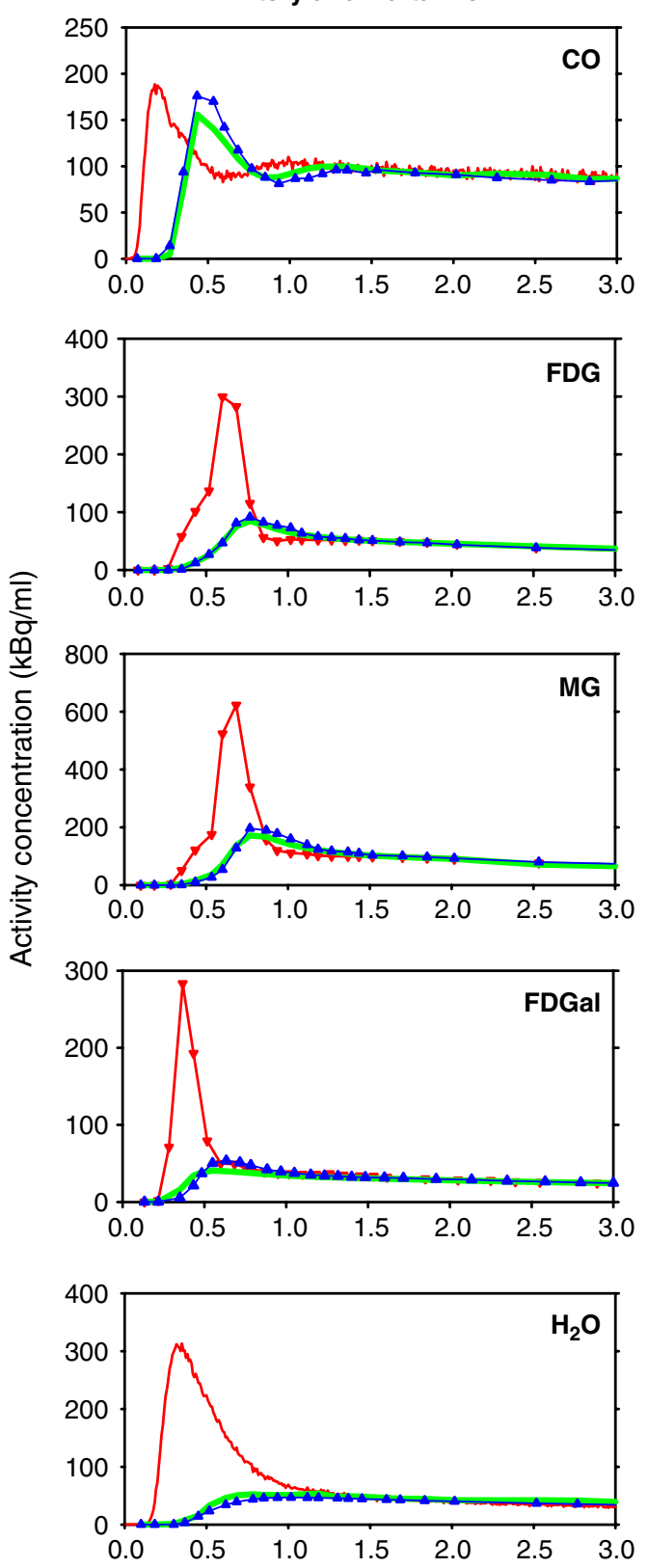

Dual Input
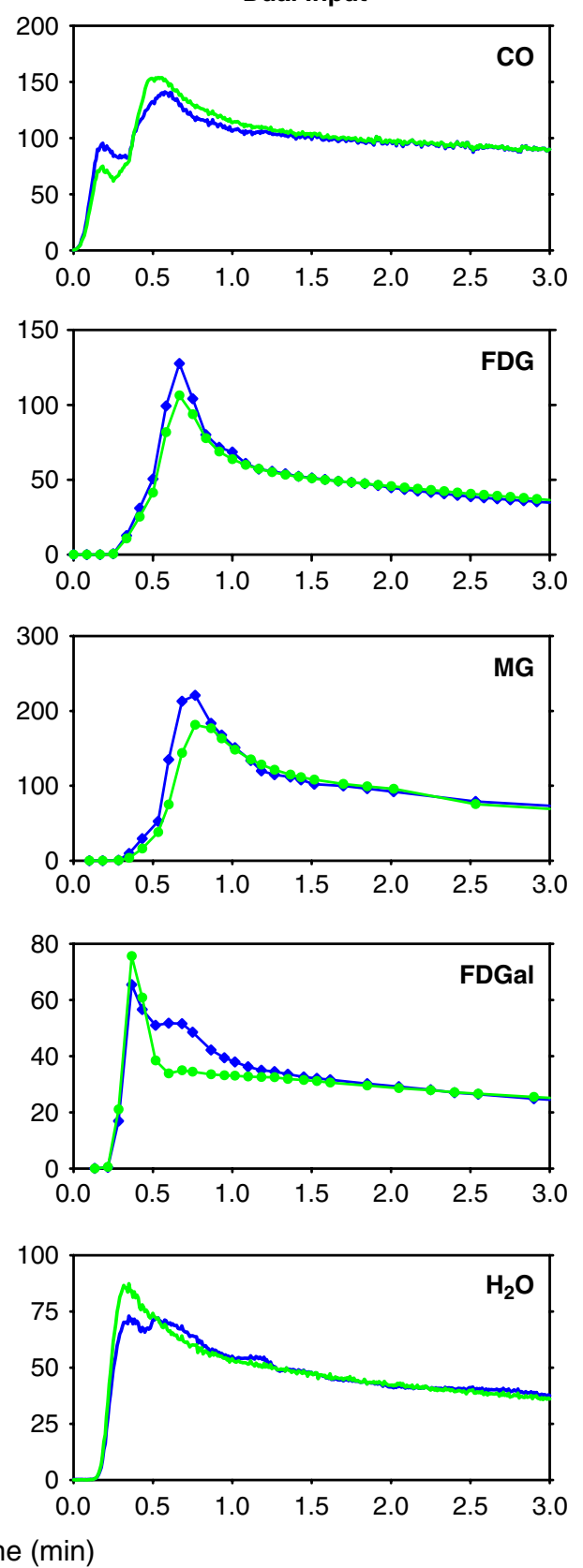

slightly over- or underestimated the peak value of $C_{\text {dual }}(t)$ more than would be expected from the good agreement between $\widetilde{C}_{\mathrm{PV}}(t)$ and $C_{\mathrm{PV}}(t)$. No systematic tendencies were apparent, probably because individual values of $f_{\mathrm{HA}}$ were used to calculate $C_{\text {dual }}(t)$, whereas the mean value $\overline{f_{\mathrm{HA}}}$ was used to calculate $\widetilde{C}_{\text {dual }}(t)$.

The error associated with using $\widetilde{C}_{\text {dual }}(t)$ rather than $C_{\text {dual }}(t)$ to estimate $K_{1}{ }^{\text {est }}$ did not differ significantly from zero with any of the tracers (Table 2). Individual estimates of $K_{1}{ }^{\text {est }}$ may, however, be biased because the individual (unknown) parameters $\beta$ and $f_{\mathrm{HA}}$ may deviate from the tracer-specific mean $\bar{\beta}$ and the mean $\overline{f_{\mathrm{HA}}}$ for all tracer administrations. As an example, Fig. 2 shows a plot of the error of $K_{1}{ }^{\text {est }}$ as a function of $\beta$ and $f_{\mathrm{HA}}$ for $\mathrm{MG}$ with $\bar{\beta}=0.5$ and $\overline{f_{\mathrm{HA}}}=0.25$. There were wide ranges of $\beta$ and $f_{\mathrm{HA}}$ values which only induce a modest error in $K_{1}{ }^{\text {est }}$. In general, $K_{1}{ }^{\text {est }}$ is overestimated when the individual $\beta$ is lower than $\bar{\beta}$ and vice versa.

\section{Discussion}

The main result of this study was that estimates of the timecourse of the model-derived $\widetilde{C}_{\text {dual }}(t)$ based on tracer-specific means of $\beta$ combined with the mean HA blood flow 
Table 1 Values of $\bar{\beta}$ (tracer-specific means of $\beta$ ) in pigs. The data are presented as means \pm SEM

\begin{tabular}{lll}
\hline Tracer & Number of tracer administrations & $\bar{\beta}(\mathrm{min})$ \\
\hline $\mathrm{CO}$ & 9 & $0.10 \pm 0.02$ \\
$\mathrm{FDG}$ & 6 & $0.50 \pm 0.05$ \\
$\mathrm{MG}$ & 6 & $0.57 \pm 0.09$ \\
$\mathrm{FDGal}$ & 10 & $0.82 \pm 0.08$ \\
$\mathrm{H}_{2} \mathrm{O}$ & 5 & $2.17 \pm 0.13$ \\
\hline
\end{tabular}

fraction, $\overline{f_{\mathrm{HA}}}$, agreed well with invasively measured $C_{\text {dual }}(t)$. The use of $\widetilde{C}_{\text {dual }}(t)$ instead of $C_{\text {dual }}(t)$ to estimate clearance of tracer from blood to liver cells, $K_{1}$, was successful for all tracers. Dual input of tracer to the liver can thus be estimated solely by sampling blood from a peripheral artery, thereby obviating the need to sample blood from the PV and perform invasive measurement of blood flow.

The tracer-specific $\beta$ (minutes) is related to the transit time of tracer through the splanchnic circulation and may be interpreted as the inverse of the most frequent rate $\left(\mathrm{min}^{-1}\right)$ by which tracer molecules enter the PV bloodstream from the intestinal arteries [14]. The study included five tracers differing widely in splanchnic mean transit time from purely intravascular $\mathrm{CO}$ to freely diffusible $\mathrm{H}_{2} \mathrm{O}$. A high mean transit time yields a high $\beta$, as reflected in the rank order of $\bar{\beta}$ for the tracers, i.e. $\mathrm{CO}<\mathrm{FDG} \approx \mathrm{MG}<\mathrm{FDGal}$ $<\mathrm{H}_{2} \mathrm{O}$ (Table 1). The low $\bar{\beta}$ for $\mathrm{CO}(0.10 \pm 0.02 \mathrm{~min})$ is in agreement with a low mean splanchnic transit time due to firm binding to erythrocytes and with the previously recorded value of $0.11 \pm 0.02 \mathrm{~min}$ in pigs [14]. $\bar{\beta}$ was significantly higher for $\mathrm{H}_{2} \mathrm{O}$ than for all the other tracers $(p<0.0001)$, in accordance with the fact that water is freely diffusible and thus has the greatest volume of distribution and slowest passage through the splanchnic circulation, i.e.

Table 2 Fractional error of model-derived clearance of tracer from blood to liver cells

\begin{tabular}{llll}
\hline Tracer & No. of studies & $K_{1}{ }^{\text {est }}$ fractional error & \\
\hline $\mathrm{CO}$ & 9 & $0.02 \pm 0.05$ & 0.71 \\
$\mathrm{FDG}$ & 6 & $0.23 \pm 0.23$ & 0.36 \\
$\mathrm{MG}$ & 6 & $-0.04 \pm 0.09$ & 0.67 \\
$\mathrm{FDGal}$ & 10 & $-0.09 \pm 0.13$ & 0.51 \\
$\mathrm{H}_{2} \mathrm{O}$ & 5 & $0.17 \pm 0.09$ & 0.16 \\
\hline
\end{tabular}

${ }^{a}$ Model-derived dual input was validated by comparing the estimated clearance of tracer from blood to liver cells, $K_{1}{ }^{\text {est }}$, with the fixed "true" clearance, $K_{1}{ }^{\text {true }} . K_{1}{ }^{\text {est }}$ was calculated by fitting a one-tissue compartmental liver kinetic model to a simulated tracer concentration time-course in liver tissue, $\widetilde{C}_{\text {liver }}(t)$, using the model-derived dual input, $\widetilde{C}_{\text {dual }}(t)$; see text for further details. The data are presented as means \pm SEM.

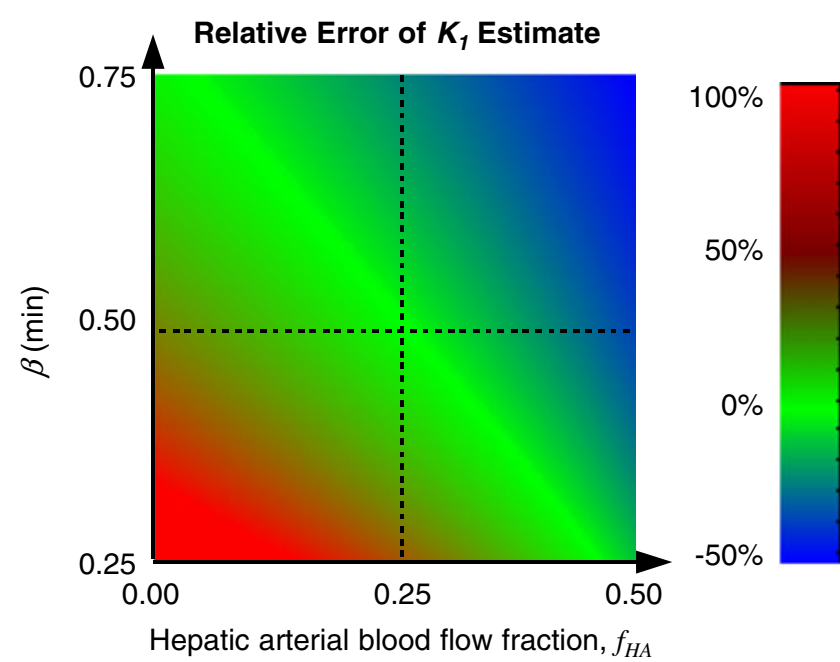

Fig. 2 Plot of the error associated with the use of model-derived dual input, $\widetilde{C}_{\text {dual }}(t)$, rather than measured dual input, $C_{\text {dual }}(t)$, (see Fig. 1 , right) for fitting hepatic kinetics. The plot provides an example with $\bar{\beta}$ equal to that for $\mathrm{MG}(0.5 \mathrm{~min})$ and $\overline{f_{\mathrm{HA}}}$ the mean of all experiments (0.25), using a simulated activity concentration time-course in liver tissue calculated according to a one-tissue compartmental model with fixed $K_{1}{ }^{\text {true }}\left(K_{1}\right.$, clearance of tracer from blood to liver cells) using $C_{\text {dual }}(t)$ as input (see text for further details). Next $K_{1}{ }^{\text {est }}$ was estimated by fitting the model to the simulated liver tissue curve using $\widetilde{C}_{\text {dual }}(t)$ as input. The relative deviation of $K_{1}{ }^{\text {est }}$ from $K_{1}{ }^{\text {true }},\left(K_{1}{ }^{\text {est }}-K_{1}{ }^{\text {true }}\right) /$ $K_{1}{ }^{\text {true }}$, illustrates how well the fixed $K_{1}{ }^{\text {true }}$ is recovered by $K_{1}{ }^{\text {est }}$, and is plotted as a function of sets of $\beta$ (tracer-specific parameter of the PV model, minutes) and $f_{\mathrm{HA}}$ (HA blood flow fraction)

a high transit time. Tracer-specific means of $\beta$, i.e. $\bar{\beta}$, could be estimated with all five tracers and the PV model adequately described the transfer of the tracers from the intestinal arteries to the $\mathrm{PV}$, thus indicating general applicability of the PV model.

The validity of the PV model relies on two assumptions: (1) no trapping of tracer in the intestines, and (2) no production of blood-born metabolites in the intestines. If one of these assumptions is violated then there is no longer a mass balance between the tracer activity concentration on the arterial side and on the PV side, and this must be taken into account, e.g. by multiplying a loss term to the impulseresponse function in Eq. 3. We did not include a loss term because all five tracers in this study allowed direct use of the PV model (Fig. 1, left). However, if other tracers are used this modification may be required. Moreover, some PET tracers, e.g. ${ }^{13} \mathrm{~N}$-ammonia, produce blood-born metabolites outside the intestines that must be taken into account when analysing dynamic PET data of the liver [20]. For such tracers the PV model should be applied separately for the tracer and the metabolites.

The mean HA flow fraction found in this study, 0.25 , is similar to the value of $0.26 \pm 0.02(n=7)$ determined in humans with no liver disease by means of ultrasound transit-time 
flow-meters [21], and of $0.25 \pm 0.02(n=8)$ determined in healthy humans by hepatic scintiangiography [22]. This indicates that a HA flow fraction of 0.25 is applicable to human studies. Likewise, the mean transit time for $\mathrm{CO}$ through the splanchnic circulation determined in pigs, $0.35 \pm$ $0.05 \mathrm{~min}$ [21], is similar to that in humans, $0.37 \pm 0.05 \mathrm{~min}$ [22]. These similarities for key physiological features are promising for translation of the present modelling to human studies, but this needs to be examined further.

A key question is to what extent will possible errors introduced by the use of the model-derived dual input, $\widetilde{C}_{\text {dual }}(t)$, rather than the measured dual input, $C_{\text {dual }}(t)$, propagate into errors of the estimated liver kinetic parameters. We examined this question by using simulated liver tissue activity concentration time-courses rather than experimental PET data, the rationale being that simulated data are not influenced by the experimental uncertainty associated with measured activity concentration timecourse in liver tissue or the possible shortcomings of compartmental models of hepatic tracer kinetics. We therefore generated a simulated activity concentration time-course in liver tissue, $\widetilde{C}_{\text {liver }}(t)$, using a one-tissue compartmental model of the hepatic tracer kinetics with fixed "true" parameters and the "correct" individually invasively measured dual input, $C_{\text {dual }}(t)$. The model was then fitted to the simulated liver tissue curve $\widetilde{C}_{\text {liver }}(t)$ using $\widetilde{C}_{\text {dual }}(t)$ to test how well the fixed "true" parameter $K_{1}$ true could be reproduced. Full kinetics of experimentally measured PET data should be analysed using kinetic models appropriate to the metabolism of the specific tracer $[1-3,18,19]$. For the evaluation of the PV model, however, all five tracers distribute flow-limited in the liver and therefore the use of the one-tissue compartmental model is relevant for the initial kinetics for all five tracers.

The finding that the mean deviation of $K_{1}{ }^{\text {est }}$ from $K_{1}^{\text {true }}$ was not significantly different from zero for any of the five tracers (Table 2) demonstrates that the modelling of the dual input yielded accurate estimates of $K_{1}$ for each group of tracer experiments. The next question is to what extent the error in $K_{1}$ for individual subjects depends on the individual (unknown) values of $\beta$ and $f_{\mathrm{HA}}$ compared with $\bar{\beta}$ and $\overline{f_{\mathrm{HA}}}$. Fig. 2 gives an example of how deviation in true individual $\beta$ and $f_{\mathrm{HA}}$ from $\overline{\mathrm{fHA}_{\mathrm{HA}}}$ and $\bar{\beta}$ propagate into errors of $K_{1}{ }^{\text {est }}$. The relationship is complicated due to correlations between the one-tissue compartmental model parameters $\left(K_{1}, k_{2}, V_{0}\right)$ and the model-derived dual-input parameters $\bar{\beta}$ and $\overline{f_{\mathrm{HA}}}$. Nevertheless, wide ranges of $\beta$ and $f_{\mathrm{HA}}$ exist that only induce a modest error in $K_{1}{ }^{\text {est }}$. However, large deviations in $\beta$ from $\bar{\beta}$ and in $f_{\mathrm{HA}}$ from $\overline{\mathrm{fA}_{\mathrm{HA}}}$ will lead to significant errors in the estimation of $K_{1}$. This emphasizes the importance of using a well-established set of $\bar{\beta}$ and $\overline{f_{\mathrm{HA}}}$ when performing kinetic analyses without PV blood samples. Thus, the model-derived dual input $\widetilde{C}_{\text {dual }}(t)$ can only be used for a group of subjects for whom estimates of $\bar{\beta}$ and $\overline{f_{\mathrm{HA}}}$ are available.

Compared to previous models [4, 7-13], our method is very robust, relying on only a single tracer-specific parameter $\bar{\beta}$. It makes no assumption about the shape of the arterial input curve, is independent of the liver kinetic model and is unaffected by possible undesirable features of the PET image-derived input such as partial volume effects and artefacts due to patient motion. The robustness of the model and the similarities between the splanchnic circulation in pigs and humans indicate the potential for transferring the method to PET studies of initial tracer kinetic processes such as blood-cell exchange and hepatic blood perfusion in liver physiology and metabolism in humans.

\section{Conclusion}

In the present study we developed a method to estimate a model-derived dual-input using the one-parametric PVmodel, a tracer-specific mean parameter $\bar{\beta}$ for the transfer of tracer from the intestinal arteries to the PV and the HA blood flow fraction $\overline{\mathrm{fA}_{\mathrm{HA}}}=0.25$. Although the five tracers examined here differ markedly in splanchnic mean transit time, the PV-model could be successfully applied to each of the tracers. Validation of the model for a wide range of tracers did not reveal any systematic deviations in parameters of the initial hepatic tracer kinetics by using the model-derived compared with the invasively measured dual-input. The study thus shows that the dual input of tracer to the liver from the HA and PV can be well approximated without the necessity to sample blood from the PV and perform invasive measurement of blood flow in the HA and PV. Our new method only requires measurement of the tracer concentration in a peripheral artery during a dynamic PET study of the liver and estimates of the mean $\overline{f_{\mathrm{HA}}}$ and tracer-specific population mean $\bar{\beta}$.

Acknowledgments This study was supported by the National Institutes of Health (R01-DK074419), the Danish Medical Research Council (09-067618), the Novo Nordisk Foundation (R121-A10313), Aase and Ejnar Danielsen's Foundation (106309), Helga and Peter Korning's Foundation, and the A. P. Møller Foundation for the Advancement of Medical Science.

The authors wish to thank Prof. Ludvik Bass, University of Queensland, Brisbane, Australia, for encouraging us to use the tracer-specific portal vein model to determine the noninvasive dualinput tracer concentration for studies of liver kinetics.

Open Access This article is distributed under the terms of the Creative Commons Attribution Noncommercial License which permits any noncommercial use, distribution, and reproduction in any medium, provided the original author(s) and source are credited. 


\section{References}

1. Munk OL, Bass L, Roelsgaard K, Bender D, Hansen SB, Keiding S. Liver kinetics of glucose analogs measured in pigs by PET: importance of dual-input blood sampling. J Nucl Med 2001;42:795-801.

2. Iozzo P, Jarvisalo MJ, Kiss J, Borra R, Naum GA, Viljanen A, et al. Quantification of liver glucose metabolism by positron emission tomography: validation study in pigs. Gastroenterology 2007;32:531-42.

3. Sørensen M, Munk OL, Mortensen FV, Olsen AK, Bender D, Bass L et al. Hepatic uptake and metabolism of galactose can be quantified in vivo by $2-[18 \mathrm{~F}]$ fluoro-2-deoxy-galactose positron emission tomography. Am J Physiol Gastrointest Liver Physiol 2008;295:G27-36.

4. Choi Y, Hawkins RA, Huang S-C, Brunken RC, Hoh CK, Messa C, et al. Evaluation of the effect of glucose ingestion and kinetic model configurations of FDG in the normal liver. J Nucl Med 1994;35:818-23.

5. Keiding S, Hansen SB, Rasmussen HH, Gee A, Kruse A, Roelsgaard K, et al. Detection of cholangiocarcinoma in primary sclerosing cholangitis by positron emission tomography. Hepatology 1998;28:700-6.

6. Prytz H, Keiding S, Björnsson E, Broomé U, Almer S, Castedal $\mathrm{M}$, et al. Dynamic FDG-PET is useful for detection of cholangiocarcinoma in patients with PSC listed for liver transplantation. Hepatology 2006;44:1572-80.

7. Slimani L, Kudomi N, Oikonen V, Jarvisalo M, Kiss J, Naum A, et al. Quantification of liver perfusion with [15O]H2O-PET and its relationship with glucose metabolism and substrate levels. J Hepatol 2000;48:974-82.

8. Brix G, Ziegler SI, Bellemann ME, Doll J, Schosser R, Lucht R, et al. Quantification of [18F]FDG uptake in the normal liver using dynamic PET: impact and modeling of the dual hepatic blood supply. J Nucl Med 2001;42:1265-73.

9. Chen S, Ho C, Feng D, Chi Z. Tracer Kinetic Modeling of 11Cacetate applied in the liver with positron emission tomography. IEEE Trans Med Imaging 2004;23:426-32.

10. Cui Y, Bai J. Comparison of parameter estimations using dualInput and arterial-input in liver kinetic studies of FDG metabolism. Proceedings of the 2005 IEEE Engineering in Medicine and Biology 27th Annual Conference Shanghai, China, 1-4 September 2005: pp. 2345-2348.

11. Kudomi N, Jarvisalo MJ, Kiss J, Borra R, Viljanen A, Viljanen T, et al. Non-invasive estimation of hepatic glucose uptake from
[18F]FDG PET images using tissue-derived input functions. Eur J Nucl Med Mol Imaging 2009;36:2014-24.

12. Kudomi N, Slimani L, Jarvisalo MJ, Kiss J, Lautamäki R, Naum GA, et al. Non-invasive estimation of hepatic blood perfusion from $\mathrm{H} 215 \mathrm{O}$ PET images using tissue-derived arterial and portal input functions. Eur J Nucl Med Mol Imaging 2008;35:1899911.

13. Taniguchi H, Oguro A, Koyama H, Masuyama M, Takahashi T. Analysis of models for quantification of arterial and portal blood flow in the human liver using PET. J Comput Assist Tomogr 1996;20:135-44.

14. Munk OL, Keiding S, Bass L. Impulse-response function of splanchnic circulation with model-independent constraints: theory and experimental validation. Am J Physiol Gastrointest Liver Physiol 2003;285:G671-G80.

15. Laustsen J, Pedersen EM, Terp K, Steinbrüchel D, Kure HH, Paulsen PK, et al. Validation of a new transit time ultrasound flowmeter in man. Eur J Vasc Endovasc Surg 1996;12:91-6.

16. Mortensen FV, Rasmussen JS, Viborg O, Laurberg S, Pedersen EM. Validation of a new transit time ultrasound flowmeter for measuring blood flow in colonic mesenteric arteries. Eur J Surg 1998;164:599-604.

17. Munk OL, Keiding S, Bass L. A method to estimate dispersion in sampling catheters and to calculate dispersion-free blood timeactivity curves. Med Phys 2008;35:3471-81.

18. Munk OL, Keiding S, Bass L. Capillaries within compartments: microvascular interpretation of dynamic positron emission tomography data. J Theor Biol 2003;225:127-41.

19. Munk OL, Bass L, Feng H, Keiding S. Determination of regional flow using intravascular PET tracers: microvascular theory and experimental validation for pig livers. J Nucl Med 2003;44:1862-70.

20. Keiding S, Sørensen M, Bender D, Munk OL, Ott P, Vilstrup H. Brain metabolism of $13 \mathrm{~N}$-ammonia during acute hepatic encephalopathy in cirrhosis measured by PET. Hepatology 2006;43:42-50. Correction in Hepatology 2006;44:1056.

21. Doi R, Inoue K, Kogire M, Sumi S, Takaori K, Suzuki T, et al. Simultaneous measurements of hepatic arterial and portal venous flows by transit time ultrasound volume flowmetry. Surg Gynecol Obstet 1988;167:65-9.

22. Fine DR, Glasser D, Hildebrandt D, Esser J, Lurie RE, Chetty N. An anatomic and physiological model of hepatic vascular system. J Appl Physiol 1995;79:1008-26. 